\title{
Pediatric Vaccine Hesitancy and the Utilization of Antibody Measurements: A Novel Strategy with Implications for COVID 19
}

\author{
Amrita Dosanjh (D) ${ }^{1,2}$ \\ 'Pediatric Respiratory, San Diego, CA, \\ USA; ${ }^{2}$ Dove Medical Press, Auckland, \\ New Zealand
}

Correspondence: Amrita Dosanjh Pediatric Respiratory, San Diego, CA, USA

Email pulmdI@gmail.com

\begin{abstract}
Vaccine hesitancy is a well researched area with implications for both public health and the health of children and their families The factors leading to vaccine hesitancy are often complex and involve fear of the healthcare system and the process of vaccine development, cultural viewpoints and experiences. Pediatric patients often rely on parental guidance and decision making, and this may result in a lack of immunization for some children. The availability of the COVID 19 vaccine has been widely anticipated, yet not all individuals will seek the vaccine. Once vaccines are available for children under the age of 16 years, this long-standing pediatric management issue may again emerge and impact public health. The clinical trial efficacy and safety data for children and adolescents less than 16 years of age are not yet available. A traditional approach is to discuss the concerns of the parent in relationship to presentation and review of American Association of Pediatrics (AAP) and CDC guidelines in the framework of medical and scientific explanations. This includes the presentation of efficacy and safety data. Therefore, the use of labbased antibody testing adds scientific evidence and emphasizes the need for vaccination against SARS CoV-2 and other pathogens. The purpose of this commentary is to propose lab-based testing as a potential adjunctive strategy in addressing this public health concern. Further study of a pediatric population is required to assess the impact of the selective use of lab-based testing in improving vaccination rates among a pediatric population.
\end{abstract}

Keywords: immunization, antibody, vaccine

\section{Introduction}

Vaccine hesitancy refers to indecision, for a variety of reasons, in the process of consenting to have a child vaccinated. Vaccine hesitancy is a complex process and occurs for numerous reasons and rationalizations. A more narrow definition will be used for this commentary, and vaccine hesitancy will be defined as refusal to vaccinate despite adequate access, without barriers related to socio-economic status or religious beliefs.

The American Academy of Pediatrics (AAP) surveyed pediatricians in 2006 and 2013. The rate of increase during this 7-year time period in the $\%$ of respondents refusing vaccination increased from $9.1 \%$ to $16.7 \%{ }^{1}$ During this time period, a measles outbreak in Europe promoted an investigation of the pattern of vaccination. Among the 1141 patients, mean age 86 months, in a cross-sectional study, $6.3 \%$ had incomplete vaccination against the measles virus, and $15.9 \%$ had no vaccination. The authors attributed the continued outbreaks of measles in Europe to low vaccination rates. ${ }^{2}$ This pattern of disease outbreaks and low immunization rates also applies to other preventable diseases. ${ }^{3,4}$ In another study, infants not 
vaccinated against hepatitis $B$ at birth had lower vaccination rates later in life to childhood vaccines. ${ }^{5}$ This study emphasizes early discussion and resolution of parental concerns. There are many potential psychological, contextual, socio-demographic and geographic barriers which were analyzed by clustering of conceptual frameworks. ${ }^{4}$

\section{Parental Education and Informed Decision Making}

The clinical encounter may not be sufficient to address parental concerns, which may result in an increased public health risk of communicable disease. The use of evidencebased strategies may be helpful in providing quantitative information, which may be presented visually to the parent, as proof that their child has inadequate antibody responses. Citizen and community involvement to support public health measures along with consistent ethical considerations also may be useful. ${ }^{6}$

This community-based approach emphasizes the benefits of vaccination to community members. In contrast, lab-based testing offers the patient a personal and quantitative guide to their decision making process. Lab-based testing includes either individual or antibody response panels ordered in the clinical setting to emphasize and to demonstrate the presence or lack of protection from diseases preventable with vaccination. In the context of community-based benefits to vaccine, this strategy provides individualized evidence of a lack of protection from an infection. The review of lab data is common, and the AAP screening program offers guidelines for lab-based office testing for a number of conditions. The addition of SARS CoV-2 antibody screening may be offered to parents who are hesitant to consent to the pediatric COVID 19 vaccination.

The clinical approach outlined by the AAP has cited strategies including a review of vaccine safety and potential conflicts with the pediatrician. The option of dismissing a patient from the practice is offered as one outcome of refusal of vaccination. ${ }^{1}$ Prior to this action, perhaps consideration for this novel approach to increase education and communication with the parent should be made.

\section{A Novel Approach Based on Laboratory Testing}

Laboratory testing to demonstrate responses to vaccine antigen is performed by a number of hospital-based and commercial clinical laboratories. The serum antibody titers may also be used to quantitatively demonstrate to the parent that their individual child is not protected against a particular disease. It may be stressed to the parent that their child is or is not protected against preventable disease with reference to personal laboratory results. ${ }^{3,4}$

In a study of infants not vaccinated against hepatitis $\mathrm{B}$ at birth, there were lower vaccination rates later in life to childhood vaccines. ${ }^{5}$ This study emphasizes early discussion and resolution of parental concerns. Lab-based testing includes either individual or antibody response panels which demonstrate the presence or lack of protection from diseases preventable with vaccination. Laboratory testing to demonstrate responses to vaccine antigen is performed by a number of hospital-based and commercial clinical laboratories. The serum antibody titers may also be used to quantitatively demonstrate to the parent that their individual child is or is not protected against a particular disease. It may be stressed to the parent that it is unlikely that the antibody levels will change over time without immunization, unless their child were to actually encounter the infection. This added quantitative information provided to some parents, in particular those who rely on data from vaccine safety studies to support their decision making process, may add to their understanding of the pros and cons of vaccination. ${ }^{6}$ The lab report provides a visual format to illustrate to the parent that their child has low or no immunity to a particular disease.

The drawbacks of this approach include: i) cost of the testing itself; ii) access to the testing; iii) parental refusal of all testing, including antibody testing. While the strategy may not be uniformly accepted or possible, particularly on a global scale, the quantitative evidence provided may be enough to promote the decision to vaccinate. An analysis and formal study may be performed by individual practices or in a multi-site study to further validate the impact of this strategy.

\section{The Application of Serologic Testing and Booster Vaccination Hesitancy}

There are many scientific studies demonstrating that antibody response levels are useful in the study of vaccine efficacy, duration of effectiveness and in assessing the function of B cells. ${ }^{7}$ Additionally, the issue of age of the child and waning pediatric vaccine protection is addressed by selective review of scientific findings with the parent. One example is a study of protective responses following tetanus and diphtheria vaccination. A prospective randomized phase III clinical trial showed 
that among adolescents a sustained $\geq 95 \%$ protection was demonstrated at five and ten years following immunization against diphtheria and tetanus. Standard assay techniques which are widely available were utilized. $^{7}$ The timing and age at vaccination have also been investigated, and the use of antibody testing may be informative in determining the need for a booster. The testing may indicate inadequate protection at a certain time point in the patient's course and highlights the need for a booster vaccination.

In one study, the investigators demonstrated that, among 311 infants studied at 13 months of age, 90\% of infants who received no 13 valent pneumococcal conjugate vaccine (PCV13) booster after 6 months of age had below normal levels of seroprotective antibodies. ${ }^{8,9}$ The SARS CoV-2 antibody test is widely available and provide guidance regarding past exposure and potential future risk for COVID 19 infection. ${ }^{10}$

\section{AAP Guidelines and Advice: Application of the C.A.S.E. Approach}

The inclusion of scientific data in the discussion with vaccine-hesitant parents has included the use of vaccine information, educational resources and websites. The AAP recommends that the information be personalized and that the parents' cultural viewpoint, education level and specific concerns be included when providing information. The C.A. S.E. method acknowledges parental concerns, builds knowledge and provides scientific data in the framework of providing advice to the patient based on scientific data. The science may include a review of clinical trial safety data. In one study by Gust et al, there are a number of parental attitudes regarding vaccination. Among the respondents, $28 \%$ were concerned about the safety of immunizations. While most parents consent to immunization, approximately $40 \%$ still have concerns (AAP.org). ${ }^{11,12}$

\section{Pediatric COVID I 9 Vaccination Hesitancy: A Future Concern}

During the COVID 19 pandemic, rates of childhood vaccinations fell significantly (Scientific American report, Daley, J, 2020; https://www.scientificamerican.com/arti cle/vaccinations-have-sharply-declined-nationwide-during -the-covid-19-pandemic). As parents reschedule their immunization visits, the topic of the pediatric COVID 19 vaccination may be incorporated for children older than 6 years of age.

The development of the COVID 19 vaccine for children is currently being conducted (clinicaltrials.gov), and efficacy and safety of the pediatric vaccine are yet to be determined. SARS CoV-2 antibody testing may thus be utilized to document that a child is not protected against the virus. Routine antibody testing is not necessary prior to vaccination. For some parents though, this may be an important factual result which assists them in informed decision making. One known complication of COVID 19 infection is MIS-C or multisystem inflammatory syndrome of childhood. ${ }^{13}$ By preventing COVID 19 infection, this complication may thus be avoided.

Interestingly, when a pediatric COVID 19 vaccine is available, a hesitancy pattern may emerge which is unique in contrast to vaccine hesitancy demonstrated, for example, to the annual influenza vaccination. In a study by Dror et al, COVID vaccine acceptance among nurses was less than that of the general population. ${ }^{14}$ In this study based on 1941 anonymous questionnaires, the acceptance of the pediatric COVID 19 vaccination was lower among physicians and nurses, compared to the general population of parents. In this study, while $70 \%$ of the general population planned to vaccinate their child, only $60 \%$ of physicians planned to do so. In contrast, physicians had a higher rate of acceptance of the influenza vaccination compared to the general public.

The findings described by Dror et al were obtained prior to the availability of pediatric COVID 19 vaccination clinical trial data. As more scientific data become available after clinical trial completion, the problem of vaccine hesitancy among pediatricians and their patients may change.

According to recent MMWR data, the main reasons for COVID 19 vaccine hesitancy include concerns about adverse events and safety. ${ }^{15}$ Data and scientific discussions are an important component of management discussions related to this and other pediatric vaccines. This is illustrated in Figure 1, which proposes a cyclical, repeated presentation of data, including consideration for individual antibody responses, based on lab report review with the parent (Figure 1).

\section{Conclusions}

The multiplex pediatric vaccine assay simultaneously measures antibodies to a wide variety of pediatric vaccines. The assay may be used to study the immunomodulatory effects of combination vaccines, and is 


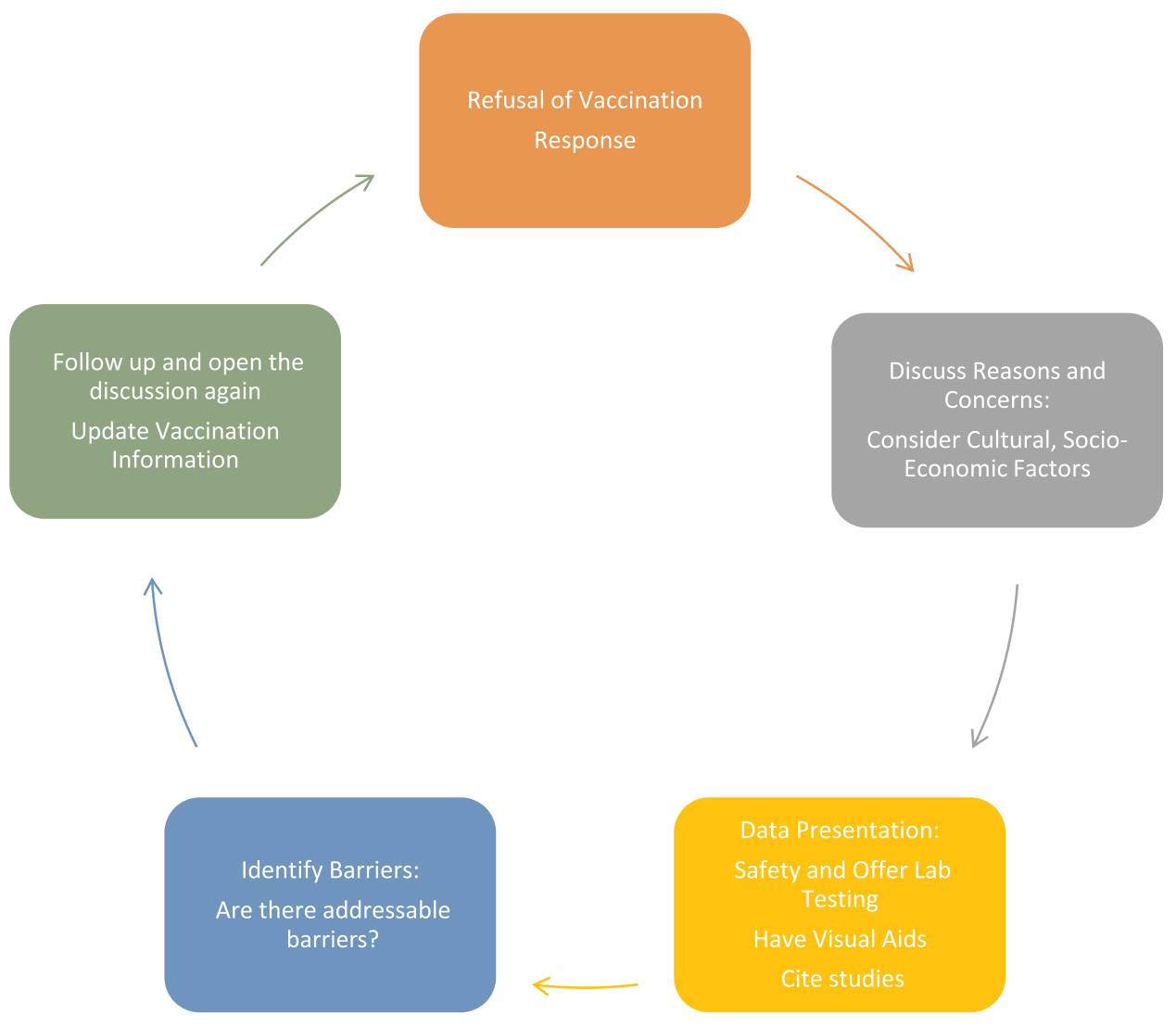

Figure I A proposed workflow for the management of pediatric vaccine hesitancy.

a highly sensitive, specific, reproducible and accurate assay. The assay is only nominally more expensive than individual antibody ELISA-based assays. ${ }^{16}$ The pediatrician may also consider individual antibody testing if the parental concern is focused on a particular vaccine. Vaccine hesitancy is now listed as one of the World Health Organization's top ten health threats. ${ }^{17}$ Thus, the use of laboratory-specific antibodies may help in the public health effort to vaccinate children against communicable disease by providing quantitative antibody titers to illustrate that the child is or is not protected if they should acquire a preventable disease. This novel approach, while not fully accessible to all parents due to healthcare access and cost, warrants further study to assess effectiveness and change in immunization rates among select populations.

\section{Disclosure}

The author is the Editor in Chief for the Journal of Asthma and Allergy for Dove Medical Press in New Zealand. The author reports no other conflicts of interest in this work.

\section{References}

1. Edwards K, Hackell J. Countering vaccine hesitancy. Pediatrics. 2016;138(3):2016-2046. doi:10.1542/peds.2016-2146

2. LoVecchio A, Cambriglia MD, Fedele MC, et al. Determinants of low measles vaccination coverage in children living in an endemic area. Eur J Pediatr. 2019;178(2):243-251. doi:10.1007/s00431-0183289-5

3. leroux-roels G, Lattanzi M, Solis CD, et al. A Phase I randomized, controlled, dose-ranging study of investigational acellular pertussis (aP) and reduced tetanus-diptheria-acellular pertussis (TdaP) booster vaccines in adults. Hum Vaccin Immunother. 2018;14(1):45-58. doi:10.1080/21645515.2017.1385686

4. Schmid P, Rauber D, Betsch C, Lidolt G, Denker ML. Barriers of influenza vaccination intention and behavior- a systemic rview of influenza vaccine hesitancy, 2005-2016. PLoS One. 2017;12(1):50. doi:10.1371/journal.pone.0170550

5. Wilson P, Taylor G, Knowles J, Blyth E, Laux J, Jhaveri R. Missed hepatitis B birth dose vaccine is a risk factor for incomplete vaccination at 19 and 24 months. J Infect. 2019;78(2):134-139. doi:10.1016/j. jinf.2018.09.014

6. Williamson L, Glaab H. Addressing vaccine hesitancy requires an ethically consistent health strategy. BMC Med Ethics. 2018;19(1):84. doi:10.1186/s12910-018-0322-1

7. Pool V, Tomovici A, Johnson DR, Greenberg DP, Decker MD. Humoral immunity 10 years after booster immunization with an adolescent and adult formulation combined tetanus, diphtheria, and component acellular pertussis vaccine in the USA. Vaccine. 2018;36 (17):2282-2287. doi:10.1016/j.vaccine.2018.03.029 
8. Zimmerman P, Perrett KP, Berbers G, Curtis N. Persistence of pneumococcal antibodies after primary immunization with a polysaccharide-protein conjugate vaccine. Arch Dis Child. 2019;104 (7):680-684.

9. Zimmerman P, Perrett K, van der Klis F, Curtis N. doi:10.1111/ imcb. 12246

10. Bartsch YC, Wang C, Zohor T, et al. Humoral signatures of protectiveand pathological SARS CoV-2 infeciton in children. Nat Med. 2021;27(3):454-462. doi:10.1038/s41591-021-01263-3

11. Jacobson RM, An Etta L, Bahta L. The C.A.S.E. approach: guidance for talking to vaccine-hesitant parents. Minnesota Medicine. 2013;96 (4):49-50.

12. Gust DA, Darling N, Kennedy A, Schwartz B. Parents with doubts about vaccines: which vaccines and reasons why. Pediatrics. 2008;122(4):718-725. doi:10.1542/peds.2007-0538

13. Rowley AH. Understanding SARS-CoV-2-related multisystem inflammatory syndrome in children. Nat Rev Immunol. 2020;20 (8):453-454. doi:10.1038/s41577-020-0367-5
14. Dror A, Eisenback N, Talber S, et al. Vaccine hesitancy: the next challenge in the fight agains COVID-19. Eur $J$ Epidemiol. 2020;35:775-779. doi:10.1007/s10654-020-00671-y

15. Nguyen K, Srivastav A, Razzaghi H, et al. COVID-19 vaccination intent, perceptions and reasons for not vaccinating among groups prioritized for early vaccination-United States, September and December 2020. MMWR. 2021;70696:217-222.

16. Iteli H, McGuire EP, Muresan P, et al. Development and application of multiplex assay for the simultaneous measurement of antibody responses elicited by common childhood vaccines. Vaccine. 2018;36 (37):5600-5608. doi:10.1016/j.vaccine.2018.07.048

17. godlee F. What should we do about vaccine hesitancy? BMJ. 2019;365:14044.

\section{Publish your work in this journal}

The Journal of Asthma and Allergy is an international, peer-reviewed open-access journal publishing original research, reports, editorials and commentaries on the following topics: Asthma; Pulmonary physiology; Asthma related clinical health; Clinical immunology and the immunological basis of disease; Pharmacological interventions and new therapies. The manuscript management system is completely online and includes a very quick and fair peer-review system, which is all easy to use. Visit http://www.dovepress.com/testimonials.php to read real quotes from published authors. 